\title{
Side-View Mirror Vibrations Induced Aerodynamically by Separating Vortices
}

\author{
Shigeru Ogawa, Taiki Kawate, Jumpei Takeda, Ittetsu Omori \\ Department of Mechanical Engineering, National Institute of Technology, Kure College, Hiroshima, Japan \\ Email: ogawa@kure-nct.ac.jp
}

Received 17 January 2016; accepted 14 March 2016; published 17 March 2016

Copyright (C) 2016 by authors and Scientific Research Publishing Inc.

This work is licensed under the Creative Commons Attribution International License (CC BY).

http://creativecommons.org/licenses/by/4.0/

(c) (i) Open Access

\section{Abstract}

While driving a car at high speed cruising, the mirror surface of side-view mirrors happens to vibrate. The vibration often leads to image blurs of objects reflected in the mirror. Once the phenomena happen, drivers cannot clearly identify the approaching vehicles from the rear. The paper aims to clarify the vibration modes of side-view mirror experimentally and to capture forces on the mirror surface induced by separating vortices around the mirror numerically. Experimental study clarified two findings. One is that the mirror has the primary natural frequencies of 25,30 and $33 \mathrm{~Hz}$. The other is that vibrations of the mirror increase in proportion to flow velocity and their frequencies have peak values at 120 and $140 \mathrm{~km} / \mathrm{h}$. The frequencies of the mirror vibration coincide completely with the primary natural frequencies. In order to capture the external forces vibrating the mirror surface, numerical study was performed by unsteady air-flow analyses. Relationships between flow velocity fluctuations close to the mirror surface and pressure fluctuations on the mirror surface were investigated. It was found that the two power spectra have peak values at the same frequency of $24.4 \mathrm{~Hz}$ at $120 \mathrm{~km} / \mathrm{h}$. This shows that flow velocity fluctuations with the frequency of $24.4 \mathrm{~Hz}$ affect directly pressure fluctuations on the mirror surface. Numerical analyses clarify that the frequencies of shedding vortices are $24.4 \mathrm{~Hz}$ at $120 \mathrm{~km} / \mathrm{h}$ and $28.3 \mathrm{~Hz}$ at 140 $\mathrm{km} / \mathrm{h}$. The frequencies of mirror vibration are very close to those of flow fluctuations. This shows that the frequencies of the mirror vibration have much to do with the frequencies of the forces induced aerodynamically by vortex shedding. Therefore it follows that image blurs at high speed cruising are caused by resonance phenomena that the mirror surface resonates with the frequencies of shedding vortices around the mirror.

\section{Keywords}

Side-View Mirror, Aerodynamic Force, Unsteady Flows, CFD Simulation, Resonance, Shedding Vortices 


\section{Introduction}

While driving a car, the mirror surface of side-view mirrors happens to vibrate. This vibration disturbs the driver to identify the approaching vehicles clearly, which has a great effect on the safety driving. The mirror vibration is defined as the image blurs induced by the vibration of the mirror surface. Once the phenomena happen, the driver look at the image blur of the object reflected in the mirror. This situation is very dangerous for the driver since the driver cannot understand clearly the approaching vehicles from the rear.

Regarding the mirror vibrations during high-speed cruising, only a few papers [1]-[3] have researched the mirror vibrations in the past. Iwai, Ishikawa, Kuroda, and Yamazaki [1] proposed a method for identifying the input power to the structure in running using the Experimental Statistical Energy Analysis (SEA) method with tracking analysis to automobile rearview mirror. This paper showed that the mirror chatter vibration in highspeed running was caused mainly by the wind and the effect of mirror chatter can be described by the input power evaluated by the Experimental SEA method. Horinouchi, Kato, Yamada, and Ninomiya [2] [3] described estimation technique for flow-induced vibrations on external rearview mirror. Unsteady flow simulation and experimental PIV method visualized the flow structure of the mirror wake. Recently, Kawate, Ogawa, and Omori [4]-[6] have so far attempted to clarify aerodynamically induced forces on the mirror surface by separating vortices, based on numerical simulation. However, in addition to the fact that there are few papers regarding mirror vibrations, these papers mentioned above have not yet revealed the mechanism of the mirror vibration caused by aerodynamically induced forces especially in terms of the relationship between input of external forces and output of vibration modes.

The present paper has the purpose of clarifying the aerodynamically induced forces on the mirror surface, and finally proposing the countermeasures for the vibration at an early stage of development in terms of the reduction of cost and weight. As a first step, the paper aims to identify dependence of the vibration modes of sideview mirror on the cruising speeds experimentally and to clarify forces on the mirror surface induced by separating vortices around the mirror numerically.

\section{Experimental Method}

The experiment was conducted in the wind tunnel. Figure 1 shows the side-view mirror where three vibration pickups (A, B, C) are placed on the mirror surface. C depicts the position of the pivot center of the mirror from which $\mathrm{A}$ and $\mathrm{B}$ is located $30 \mathrm{~mm}$ away in the $\mathrm{Y}$ and $\mathrm{Z}$ direction, respectively. The coordinate is defined in such a way that $\mathrm{X}$ axis indicates the flow direction, $\mathrm{Y}$ axis right-left ward, and $\mathrm{Z}$ axis up-down ward as shown in Figure 1. Accelerations in three directions for $\mathrm{X}, \mathrm{Y}$, and $\mathrm{Z}$ were measured by means of the vibration pickup equipped with a triaxial acceleration sensor at each point. $\mathrm{L}$ indicates representative length as $0.17 \mathrm{~m}$.

\section{Experimental Results and Discussion}

Provided that the vibration is considered as a simple harmonic motion, the shake amounts $\delta$ is described as

$$
\delta=A \sin \omega t
$$

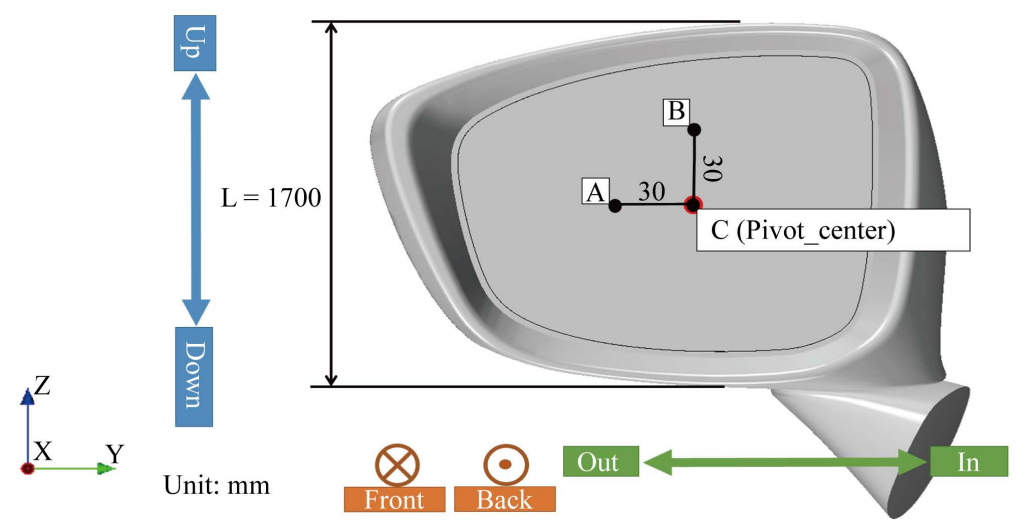

Figure 1. Location of vibration pickups of the side-view mirror. 
The acceleration $a$ on the mirror surface is given by second derivatives of $\delta$

$$
a=-A \omega^{2} \sin \omega t=-\omega^{2} \delta .
$$

Therefore, the shake amounts $\delta$ can be expressed as follows

$$
\delta=-\frac{a}{\omega^{2}}=-\frac{a}{4 \pi^{2} f^{2}}
$$

where $\omega$ is angular velocity, $f$ the vibration frequency. From this equation it is found that shake amounts are proportional to the acceleration but inversely proportional to the square of the frequency of the acceleration. It, therefore, follows that to suppress the mirror vibration it is efficient to shift the vibration frequency from lower to higher frequencies.

The vibration measurements were conducted at the wind tunnel. The mirror attached to a fixing device was placed in the test section of the wind tunnel. Figure 2 depicts the vibrating locus of the center point $C$ of the mirror surface in the virtual Y-Z plane $1000 \mathrm{~mm}$ away from the mirror at $120 \mathrm{~km} / \mathrm{s}$. X-Automobile has an image blur occurs whereas Y-automobile has no problem. There are relatively large shake amounts in X Automobile, compared with Y-automobile without shaking image.

\subsection{Natural Frequencies}

An ideal impact to a structure is a perfect impulse, which has an infinitely small duration, causing constant amplitude in the frequency domain; this would result in all modes of vibration being excited with equal energy. The impact hammer test is designed to replicate this. Natural frequencies of the mirror were measured by the impact hammer test. Figure 3 shows the coherence function of frequencies. When the values of coherence function are equal to 1 , the results are considered to have the linear relationship between the input and the output without any noise contained. Figures 4-6 show power spectra obtained by Fast Fourier Transform (FFT) analyses. The vertical axis indicates Power Spectral Density (PSD) which means energies of waves per Hz. Since the frequency zone which affects image blurs is considered to range mainly from 20 to $200 \mathrm{~Hz}$, the data obtained were, therefore, treated with high pass filter over $20 \mathrm{~Hz}$ and low pass filter below $200 \mathrm{~Hz}$ before FFT analyses. It is found that three power spectra have primary natural frequencies of 25 and $33 \mathrm{~Hz}$ in the $X$ direction whereas 25 and 30 $\mathrm{Hz}$ in the $\mathrm{Y}$ and $\mathrm{Z}$ direction. There also exist natural frequencies of higher order around $100 \mathrm{~Hz}$. These natural frequencies of the mirror are considered meaningful because their coherence functions are nearly equal to 1 .

\subsection{Resonance Frequencies}

The mirror attached to the testing apparatus, which was set in the same testing situation where the natural frequencies were measured, was immersed in the wind tunnel test section to measure how vibration frequencies of the mirror surface vary depending on the flow velocities. Figures 7-9 indicate frequency spectra of mirror surface
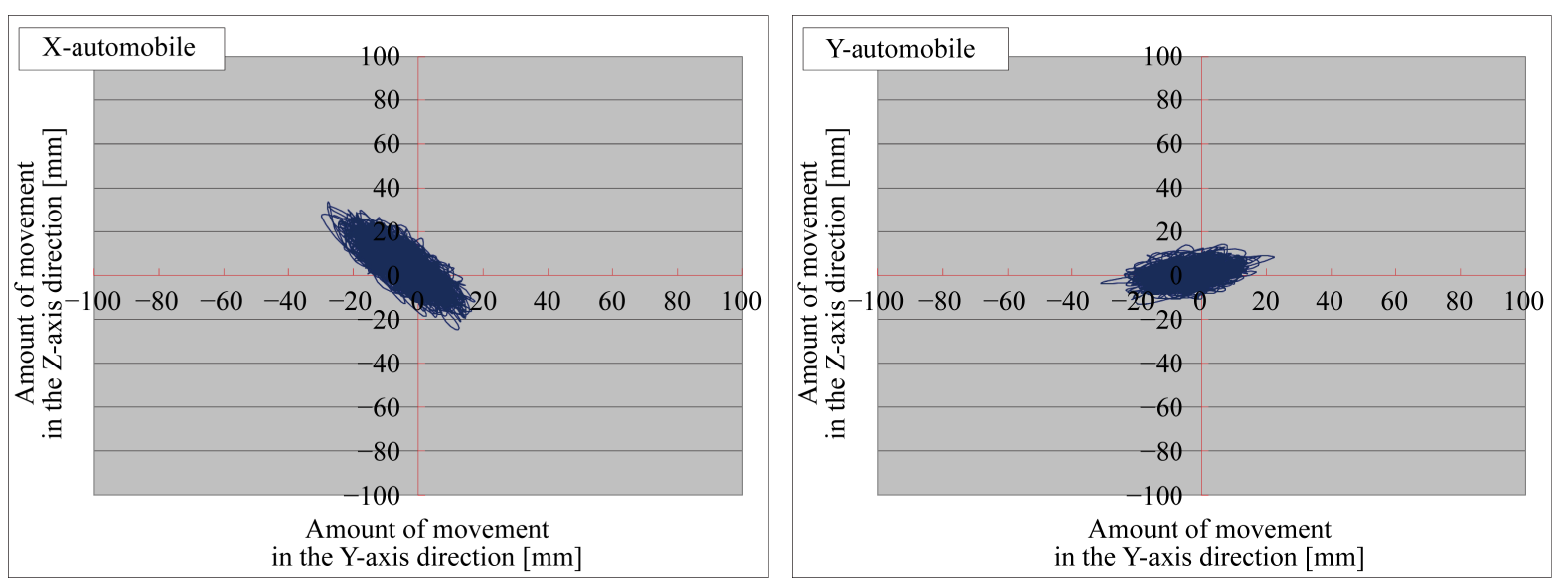

Figure 2. Vibrating locus of the center point of the mirror surface in the virtual Y-Z plane $1000 \mathrm{~mm}$ away from the mirror surface at $120 \mathrm{~km} / \mathrm{h}$. 


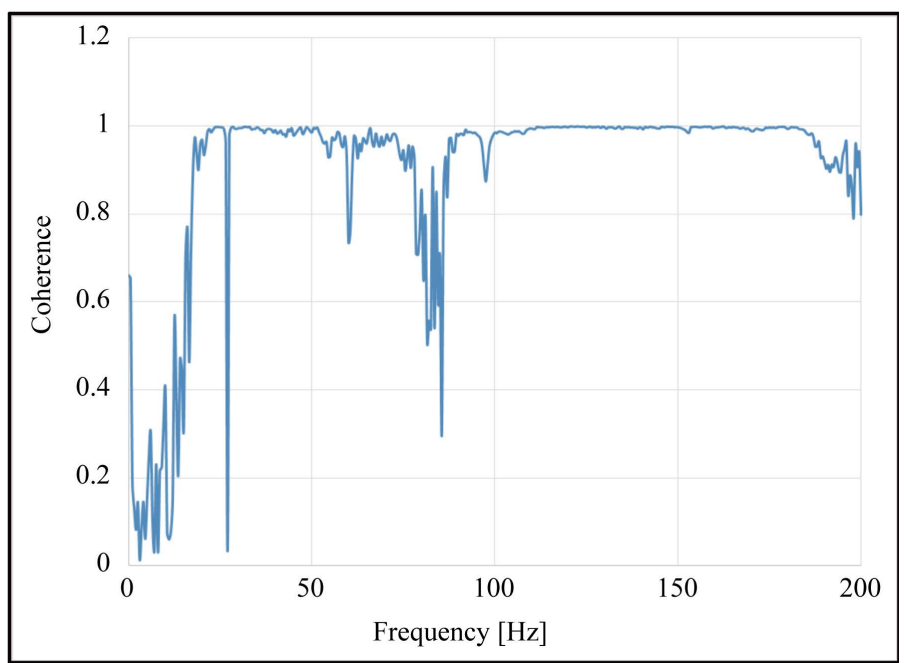

Figure 3. Coherence function of frequencies.

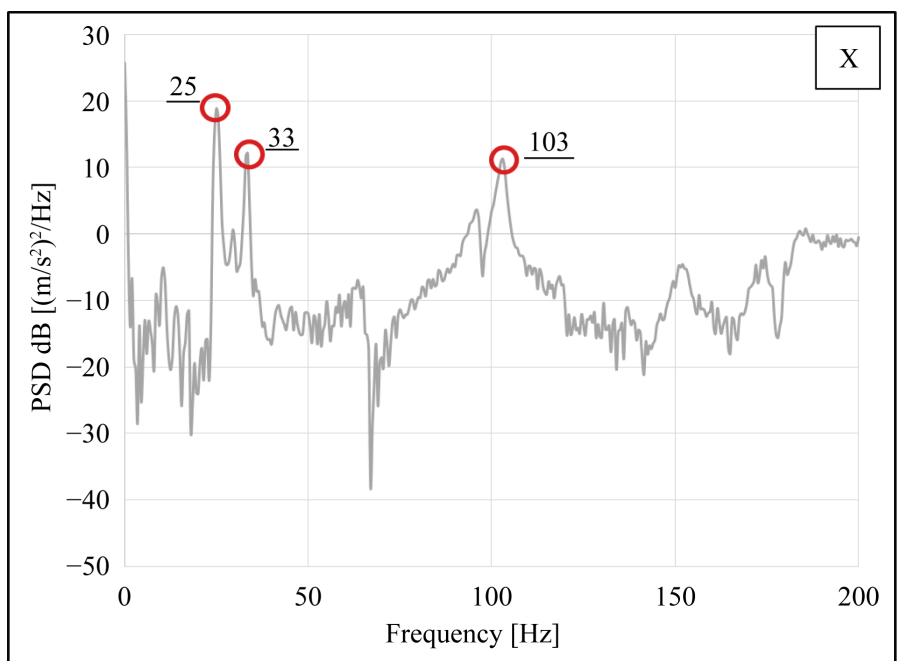

Figure 4. Relationship between power spectral density and natural frequencies in the X direction.

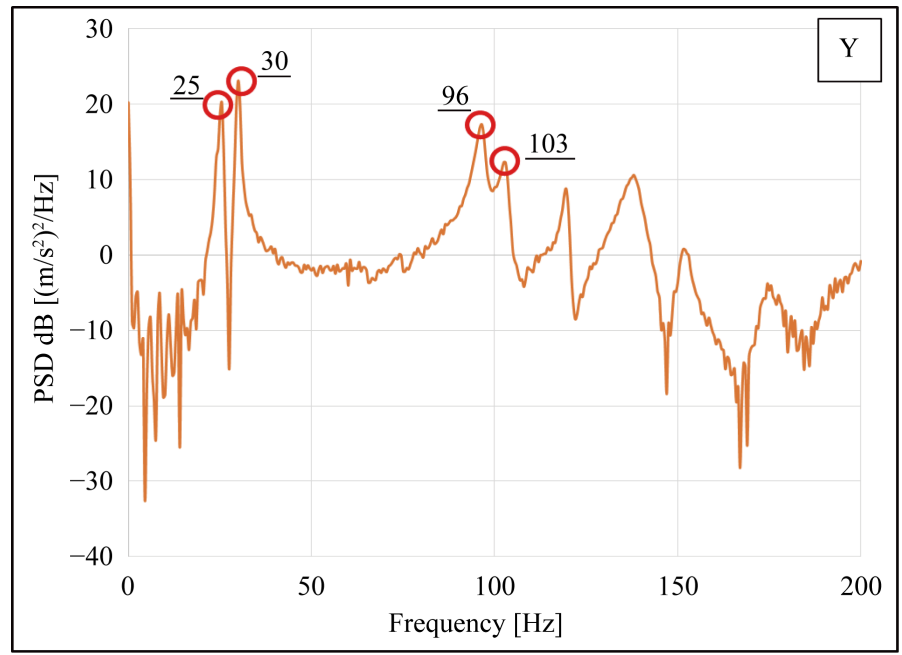

Figure 5. Relationship between power spectral density and natural frequency in the Y direction. 


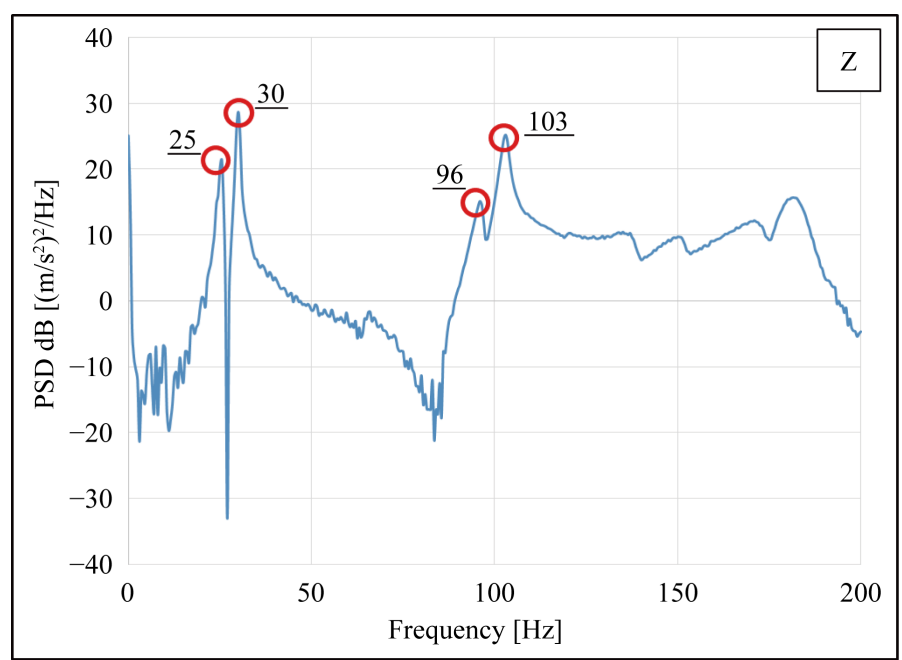

Figure 6. Relationship between power spectral density and natural frequency in the $\mathrm{Z}$ direction.

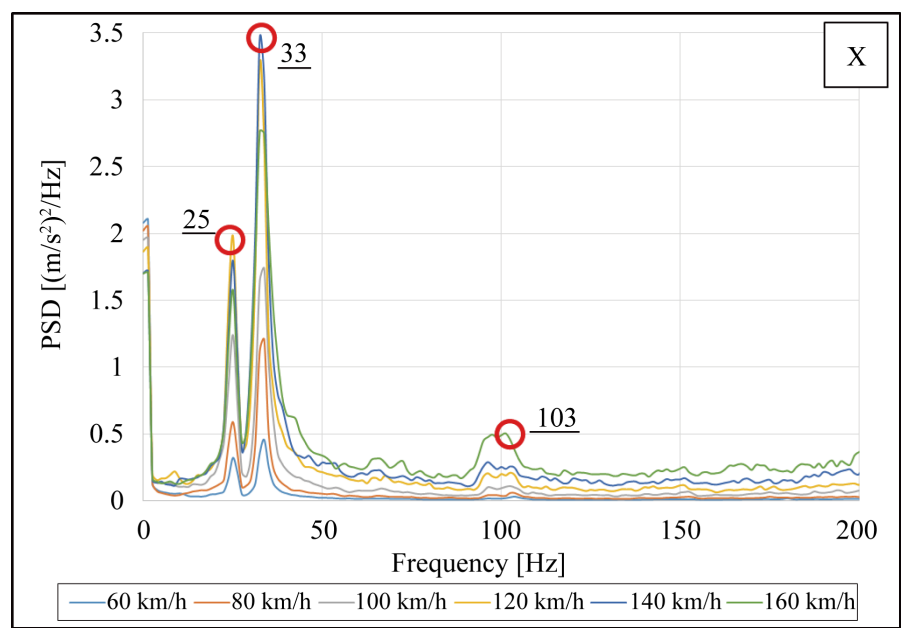

Figure 7. Frequency spectra of mirror surface vibrations in the streamwise direction for flow velocities ranging from $60 \mathrm{~km} / \mathrm{h}$ to $160 \mathrm{~km} / \mathrm{h}$ at every $20 \mathrm{~km} / \mathrm{h}$ steps.

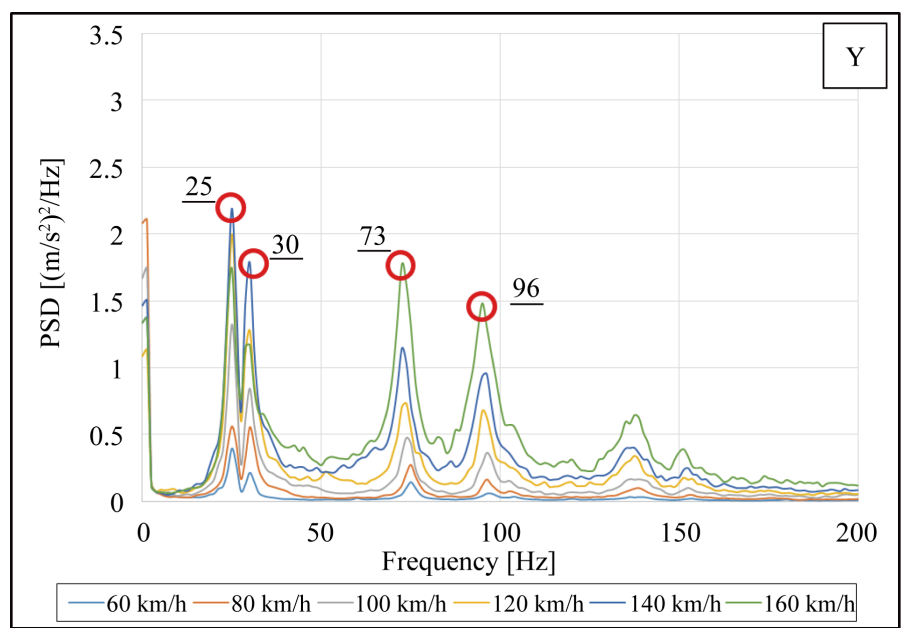

Figure 8. Frequency specra of mirror surface vibrations in the right-leftward direction for flow velocities ranging from $60 \mathrm{~km} / \mathrm{h}$ to $160 \mathrm{~km} / \mathrm{h}$ at every $20 \mathrm{~km} / \mathrm{h}$ steps. 
vibrations in $\mathrm{X}, \mathrm{Y}$ and $\mathrm{Z}$ direction, respectively. The frequency spectra were measured for the flow velocities from $60 \mathrm{~km} / \mathrm{h}$ to $160 \mathrm{~km} / \mathrm{h}$ at every $20 \mathrm{~km} / \mathrm{h}$ steps. The results show that all the vibration accelerations have the peaks at $(25,33 \mathrm{~Hz}),(25,30 \mathrm{~Hz})$, and $(25,30 \mathrm{~Hz})$ in the $\mathrm{X}, \mathrm{Y}$ and $\mathrm{Z}$ direction respectively. These vibration accelerations coincide completely with the primary natural frequencies shown in Figures 4-6. Therefore it follows that the surface of the mirror is resonating with the same frequencies as the primary natural frequencies.

Figure 10 shows overall accelerations (O.A.) are obtained as the root mean square of the accelerations in the each direction during the measuring period. It is found that overall accelerations increase in proportion to flow velocities and the streamwise acceleration in the $\mathrm{X}$ direction is the largest of the three accelerations for velocity zone measured whereas the accelerations in $\mathrm{Y}$ and $\mathrm{Z}$ direction are almost the same. Figure 11 indicates that how power spectra of the mirror surface vibrations in the $\mathrm{X}$ direction change with flow velocities. Power spectra of the frequency $25 \mathrm{~Hz}$ have the peak value at $120 \mathrm{~km} / \mathrm{h}$ and that of $33 \mathrm{~Hz}$ peaks at higher flow velocity of 140 $\mathrm{km} / \mathrm{h}$. On the other hand, Figure 12 and Figure 13 show that power spectra in the $\mathrm{Y}$ and $\mathrm{Z}$ direction for 25 and $30 \mathrm{~Hz}$ are quite similar to those in X direction with the peaks at $140 \mathrm{~km} / \mathrm{h}$. With respect to higher order frequencies such as $96 \mathrm{~Hz}, 103 \mathrm{~Hz}$, their power spectra are increasing in proportion to flow velocity. The vibration frequencies of the mirror surface shift from the lower to the higher resonance frequency. This may be caused by shedding vortices from the mirror because their frequencies are proportional to flow velocity.

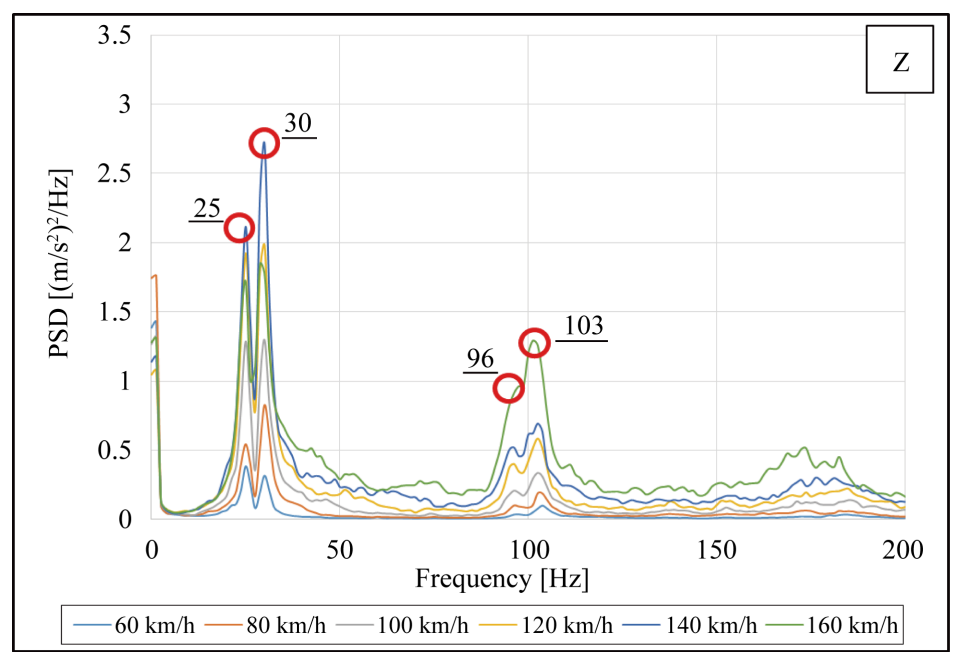

Figure 9. Frequency specra of mirror surface vibrations in the up-downward direction for flow velocities ranging from $60 \mathrm{~km} / \mathrm{h}$ to $160 \mathrm{~km} / \mathrm{h}$ at every $20 \mathrm{~km} / \mathrm{h}$ steps.

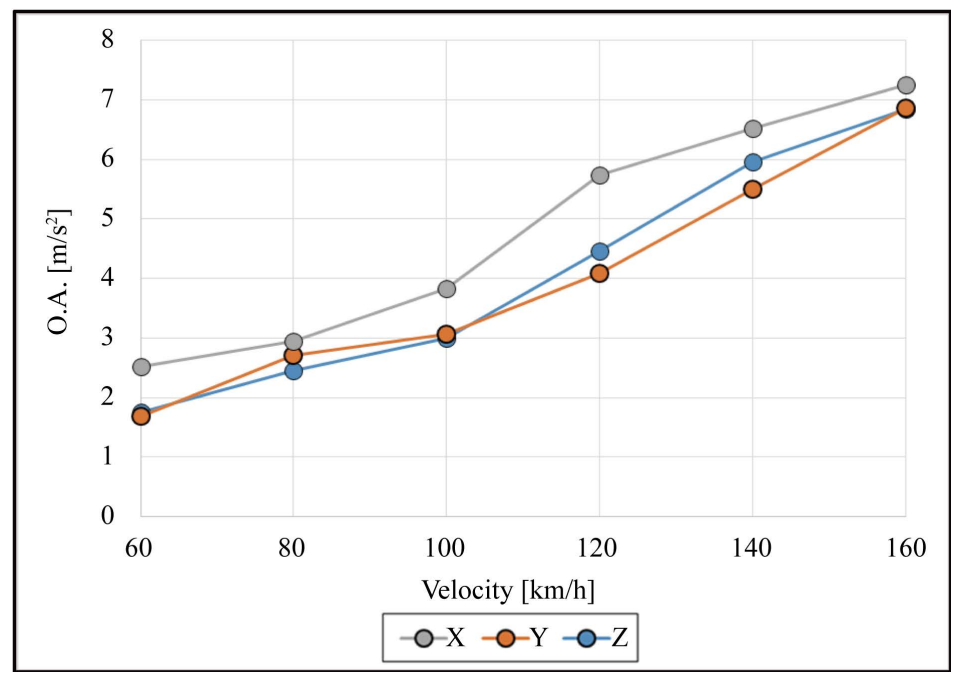

Figure 10. Dependance of overall acceleration for mirror surface on flow velocities. 


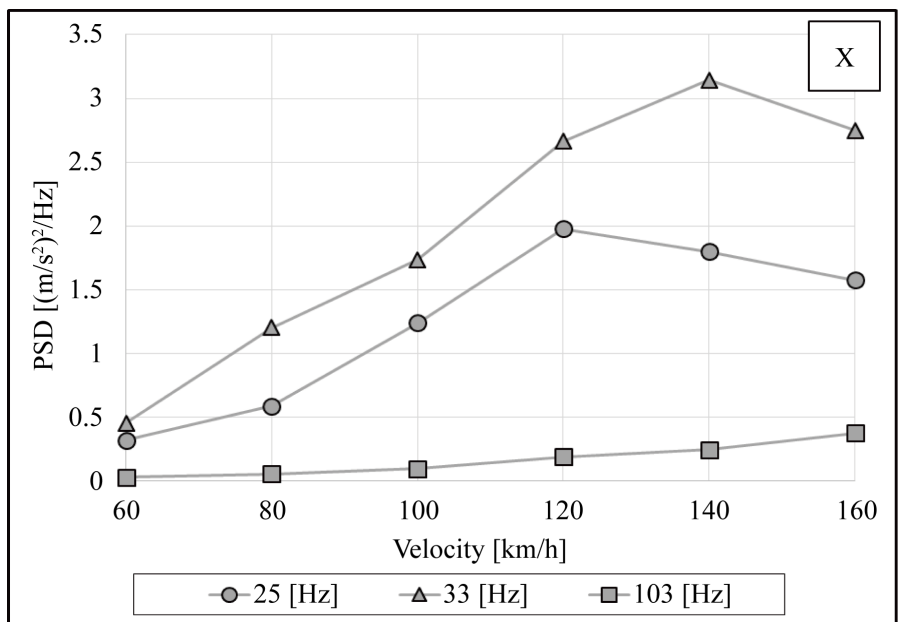

Figure 11. Dependance of power spectra of the mirror surface vibrations on flow velocities in the $\mathrm{X}$ direction.

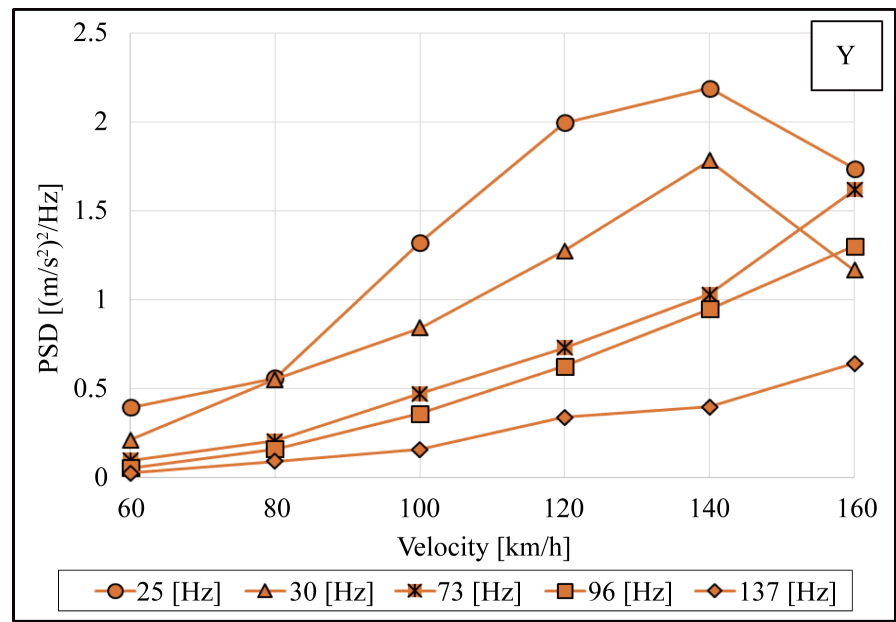

Figure 12. Dependance of overall acceleration for mirror surface on flow velocities in the $\mathrm{Y}$ direction.

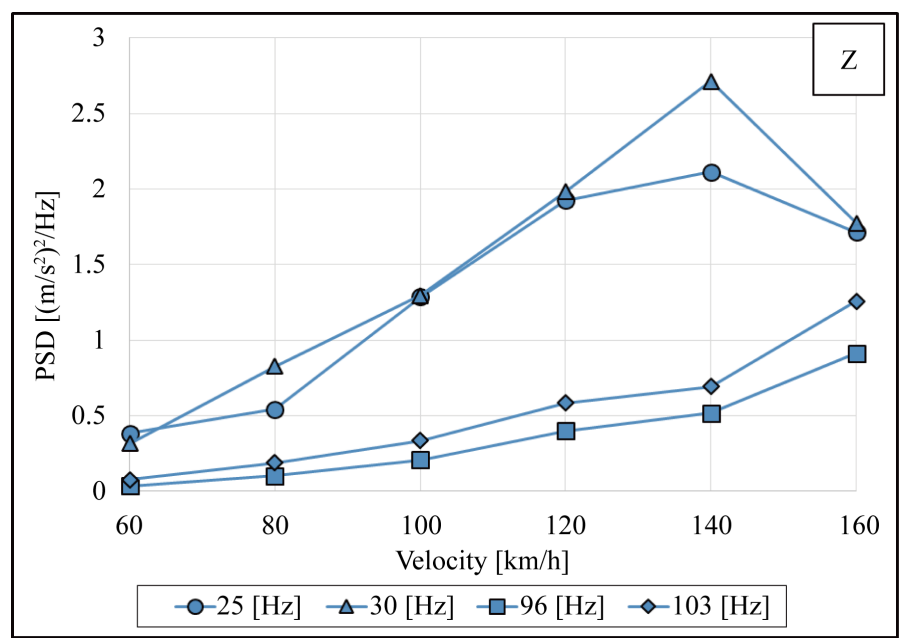

Figure 13. Dependance of power spectra of the mirror surface vibrations on flow velocities in the $\mathrm{Z}$ direction. 


\section{Numerical Analysis Method}

The study uses the software STAR-CCM+ with software V9.04.009 (for Windows 64).

\subsection{Mesh Generation Method}

Figure 14 shows the numerical wind tunnel. The scale of the numerical wind tunnel is $600 \mathrm{~mm} \times 600 \mathrm{~mm} \times$ $1200 \mathrm{~mm}$. There is a side-view mirror in the numerical wind tunnel. The total mesh number is approximately 5 million points, and mesh size close to side-view mirror becomes smaller and minimum size is $0.3 \mathrm{~mm}$. In generating mesh, prism layer mesher method was employed. This meshing method was used to optimize the mesh size in the boundary layer as shown in Figure 15. The prism layer mesh model uses a core volume mesh to generate orthogonal prismatic cells next to wall surfaces or boundaries. This layer of cells is necessary to improve the accuracy of the flow solution. The prism layer mesh is defined in terms of its thickness, the number of cell layers, and the size distribution of the layers. In this study, the thickness of the prism layer was determined so that the value of $Y_{+}$shown in Figure 16 can be 30 or less for accuracy. As a result, the thickness of prism layer is $3 \mathrm{~mm}$ and the number of cell layer is 5 and the mesh size is $0.5 \mathrm{~mm}$ closest to the model surface.

\subsection{Numerical Calculation Method}

The present study was performed by unsteady airflow analysis. Reynolds Averaged Navier-Stokes (RANS) Simulation was employed as the turbulence model for the steady-state analysis. K- $\omega$ model was used as eddy viscosity model. Flow data obtained by steady-state analysis were employed as initial values for unsteady analysis for reduction of the calculation time and for higher accuracy. Detached Eddy Simulation (DES) was used as turbulence model for the unsteady analysis and SST (Menter) K- $\omega$ model for eddy viscosity model.

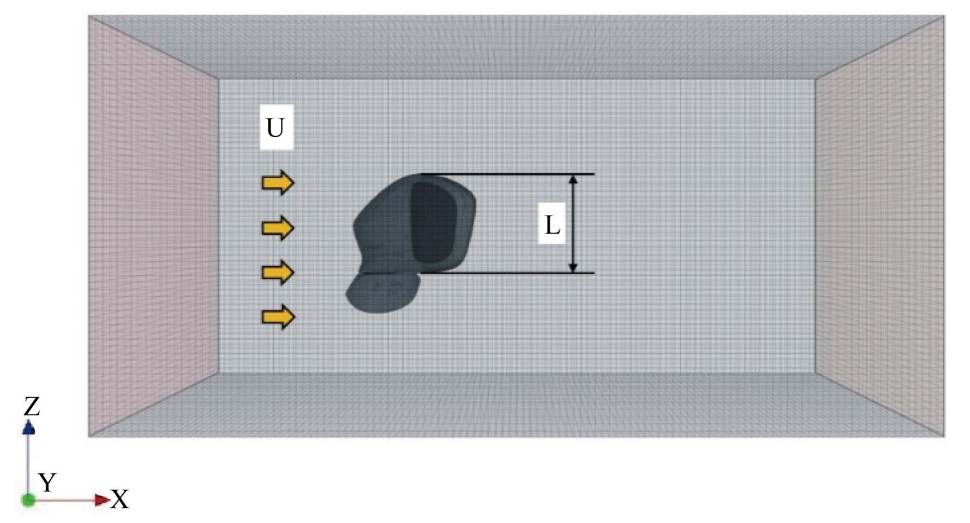

Figure 14. Numerical wind tunnel and the side-view mirror.

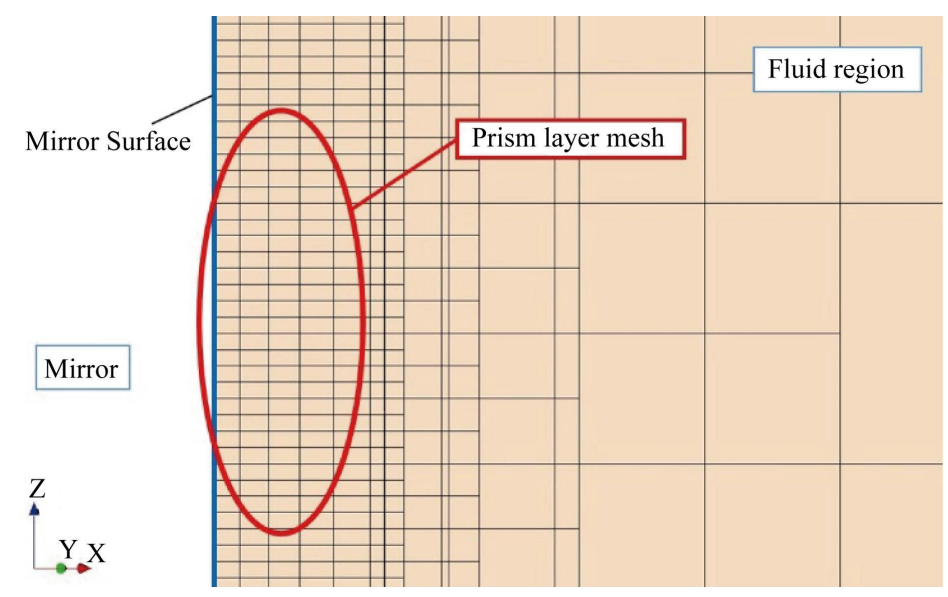

Figure 15. Prism layer mesh around the side-view mirror surface. 


\subsection{Calculation Parameter}

The side-view mirror model is no gap between the mirror and the casing, and the mirror surface is regarded as a rigid body. For the steady state analysis, the maximum step number is 3000. For the unsteady calculation, the internal iteration number is 10 , and maximum step number is 1024 , and time step is $\Delta=0.001 \mathrm{~s}$. The airflow velocity is set to $120 \mathrm{~km} / \mathrm{h}(33.3 \mathrm{~m} / \mathrm{s})$. Reynolds number is defined in Equation (2), and results in $R e=3.74 \times$ $10^{5}$, using $U=33.3 \mathrm{~m} / \mathrm{s}, L=0.17 \mathrm{~m}$, and $v=1.512 \times 10^{-5} \mathrm{~m}^{2} / \mathrm{s}$.

$$
R_{e}=\frac{U L}{v} .
$$

\section{Numerical Results and Discussion}

Figure 17 shows the time-averaged velocity distribution. The cross-section of the mirror is located at the mirror center perpendicular to the ground. The airflows are accelerated before separating at the mirror casing and after their separation negative flow velocity is generated in the vicinity of the mirror surface. Since the negative velocity indicates that the flow direction is opposite to the uniform airflow direction, it is assumed that there exist vortices generated around the mirror surface.

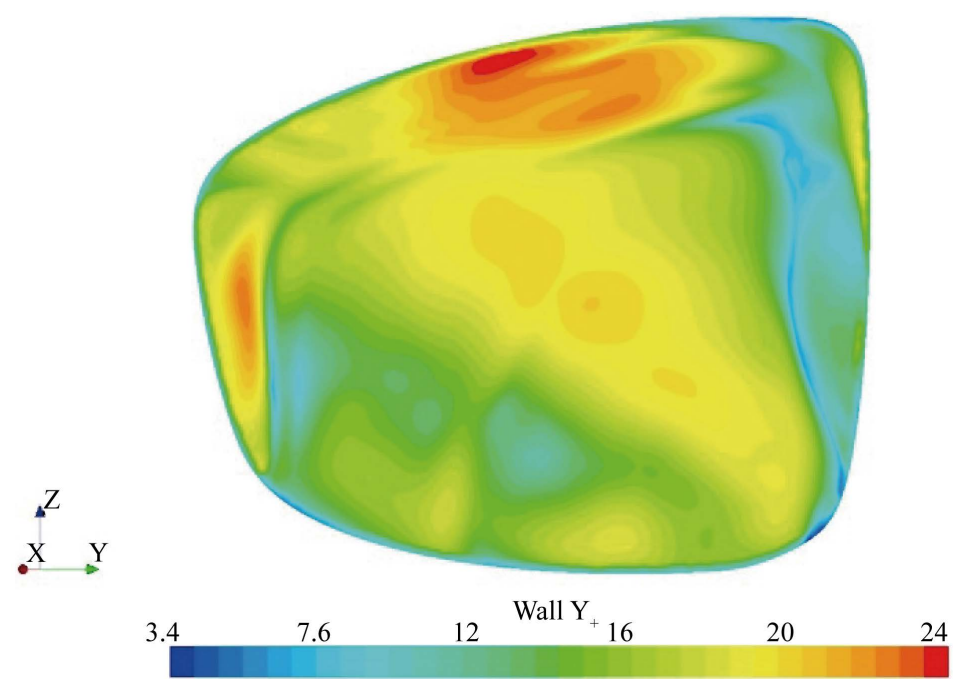

Figure 16. Wall $Y_{+}$distribution on the mirror surface.

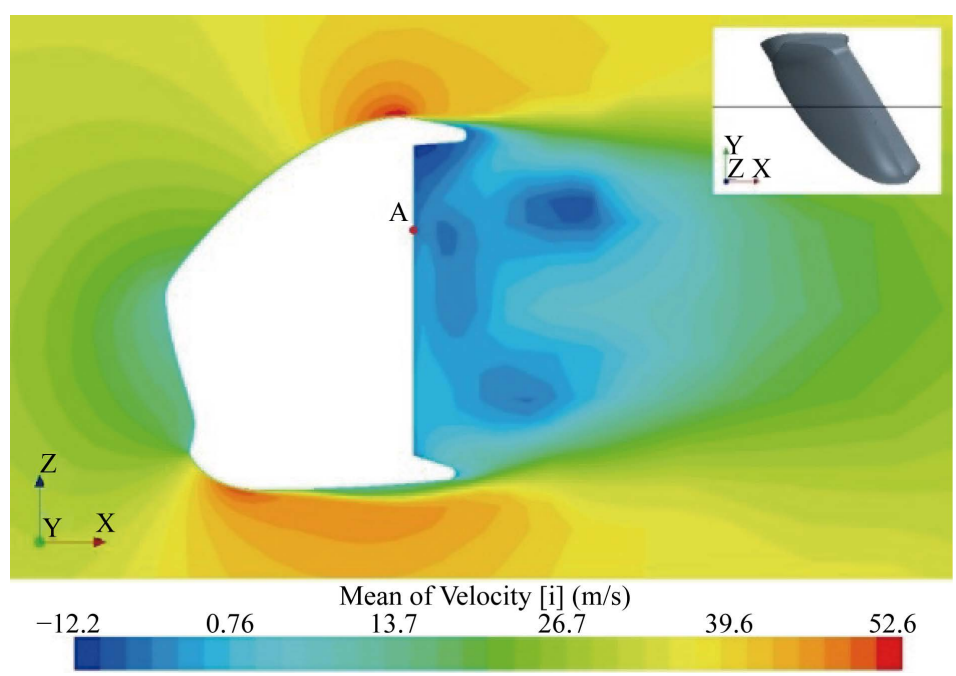

Figure 17. Time-averaged velocity distribution around the side-view mirror. 
Flow field around the mirror was investigated. Figure 18 shows the time averaged distribution in terms of velocity and vorticity at the same cross section as described in Figure 17. The arrows in the figure indicate the magnitude and direction of the velocity vector. The vorticity is defined as Equation (3) to Equation (6) with respect to $X, Y, Z$ coordinate axes and velocity vector $U(\boldsymbol{u}, \boldsymbol{v}, \boldsymbol{w})$. Three components $\omega_{i}$, $\omega_{j}$, and $\omega_{k}$ express the vorticity in $\mathrm{X}, \mathrm{Y}, \mathrm{Z}$ axial directions respectively. Figure 18 shows the $\omega_{j}$ component.

$$
\begin{aligned}
& \boldsymbol{\omega}=\nabla \cdot \boldsymbol{U} \\
& \omega_{i}=\frac{\partial \boldsymbol{w}}{\partial Y}-\frac{\partial \boldsymbol{v}}{\partial Z} \\
& \omega_{j}=\frac{\partial \boldsymbol{u}}{\partial Z}-\frac{\partial \boldsymbol{w}}{\partial X} \\
& \omega_{k}=\frac{\partial \boldsymbol{v}}{\partial X}-\frac{\partial \boldsymbol{u}}{\partial Y} .
\end{aligned}
$$

It is found that much stronger vortices are generated especially at the top and the bottom of the side-view mirror, and in vicinity of the mirror surface. There exists a pair of vortices whose rotational directions are opposite. It is also recognized that vortices with stronger vorticity exist in the vicinity of the point $\boldsymbol{A}$ on the mirror surface. The point $\boldsymbol{A}$ is selected as the place where there exist the largest accelerations on the mirror surface. Since higher vorticity is located close to the mirror surface, unsteady motions of the vortices seem to have a greater effect on the mirror surface.

In order to evaluate quantitatively the magnitude of the pressure operating on the mirror surface, pressure coefficients defined in Equation (7) is introduced as an indicator of pressure.

$$
C_{p}=\frac{P_{m}-P_{\infty}}{(1 / 2) \rho U_{\infty}^{2}}
$$

where $P_{m}$ is pressure of measurement points on the mirror surface, $P_{\infty}$ pressure in the uniform flow, $\rho$ density of airflows, $U_{\infty}$ flow velocity in the uniform flow.

Figure 19 shows the time-averaged pressure coefficient distribution on the mirror surface. Since $P_{m}$ is pressure on the mirror surface, negative $C_{p}$ means that the pressure of the entire mirror surface is lower than that of uniform airflows. It, therefore, follows that the mirror surface is always being dragged towards the vortices over

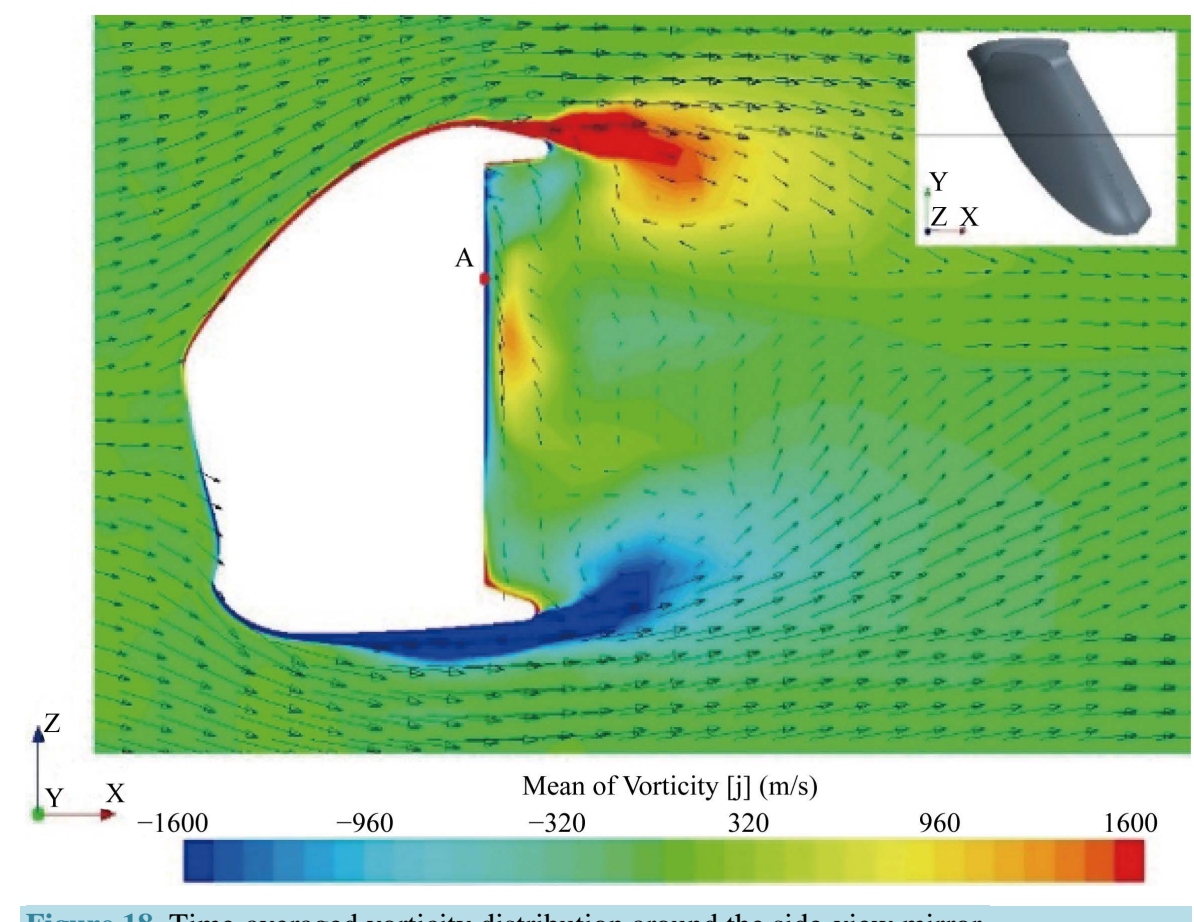

Figure 18. Time-averaged vorticity distribution around the side-view mirror. 
the mirror surface. It is found that there is much higher negative pressure in the dark blue-colored region where the point $\boldsymbol{A}$ is located.

Figure 20 shows the time-averaged force distribution on the mirror surface. The higher negative pressure shown in bright yellow distributes from the lower right side to the upper side in the vicinity of the point $\boldsymbol{A}$. It is found that the forces operating on the yellow-colored region on the right are about five times greater than those on the blue-colored one on the left. Since even time-averaged forces on the mirror have greatly different distribution, it is considered that for unsteady airflows the forces operating on the entire mirror surface could be aerodynamically induced more widely and rapidly. This unsteady and greatly different force distribution on the mirror surface can cause mirror vibration problem during high speed driving.

As mentioned above, it was found that separation vortices cause the widely different forces on the mirror surface. The characteristics of separation vortices, therefore, will be clarified in terms of frequency analysis by FFT analyses. The separation vortices will be related to the pressure fluctuation on the mirror surface. Figure 21 shows the relationship between flow velocity over the mirror surface and pressure on the mirror surface. Flow velocity is measured numerically at the point $\boldsymbol{P} 15 \mathrm{~mm}$ away in the $\mathrm{X}$ axis direction from the pressure measuring point $\boldsymbol{A}$ on the mirror surface.

Figure 22 shows the velocity fluctuations at the point $\boldsymbol{P}$ and pressure fluctuations at the point $\boldsymbol{A}$. With respect

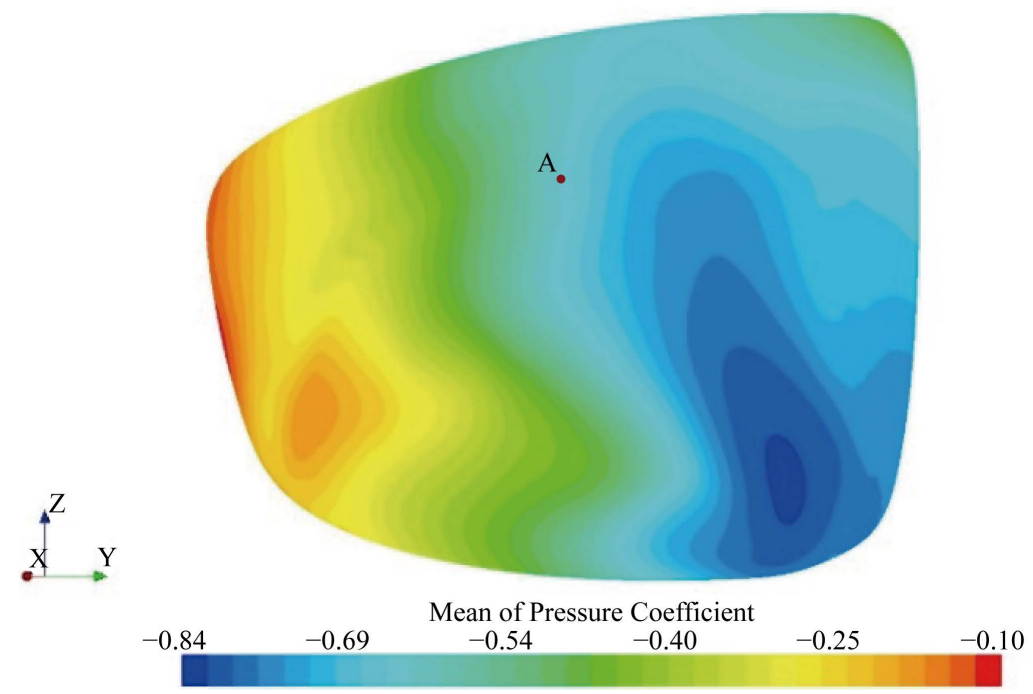

Figure 19. Pressure coefficient distribution on the mirror surface.

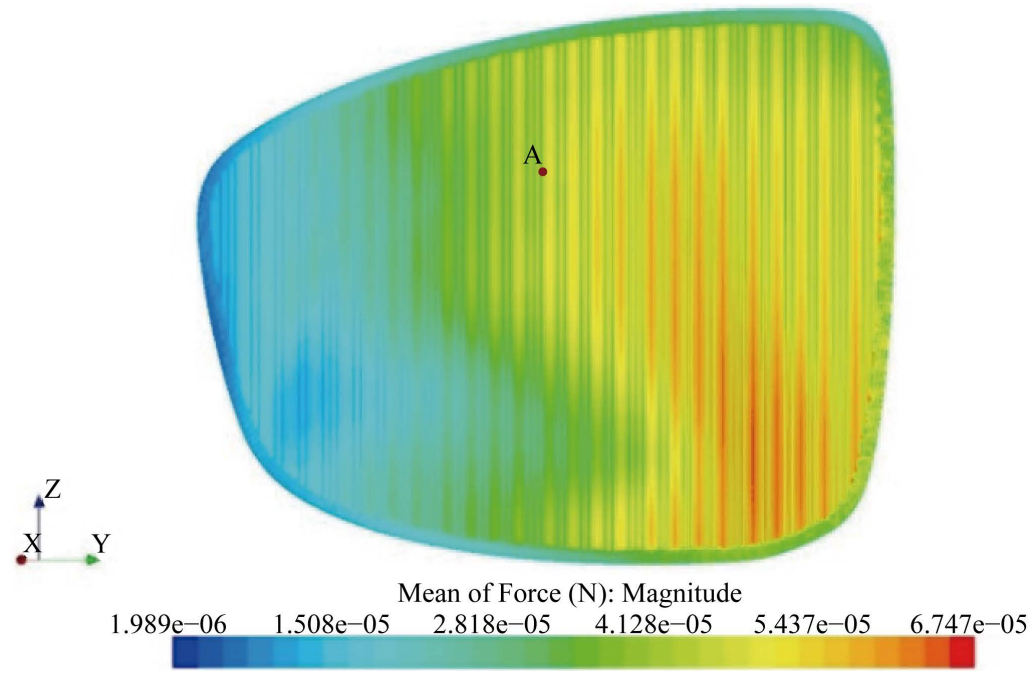

Figure 20. Time-averaged force distribution on the mirror surface. 
to the velocity fluctuations obtained at the point $\boldsymbol{P}$, they reflect the existence of vortices. This is because the flow velocity in the $\mathrm{X}$ axis direction at the point $\boldsymbol{P}$ has negative values, and maximum amplitude of the flow velocity is fluctuating up to about $11 \mathrm{~m} / \mathrm{s}$, which account for one third of uniform flow velocity $120 \mathrm{~km} / \mathrm{h}(33.3 \mathrm{~m} / \mathrm{s})$. Pressure fluctuations at the point $\boldsymbol{A}$ occurred at certain retarded time, compared with flow velocity fluctuations.

Figure 23 shows power spectral density spectra obtained by FFT method at the point $\boldsymbol{A}$ and point $\boldsymbol{P}$. In FFT method, the amplitude function was used as the power spectral density function, and the Hamming window was employed as the window function. It is found that the two power spectra have peak values at the frequency of 24.4 Hz although the left vertical axis has power spectra of velocity whereas the right axis has those of pressure. It therefore follows that separation vortices separating at the frequency of $24.4 \mathrm{~Hz}$ have an influence on pressure fluctuations on the mirror surface.

Generally speaking, the frequency $f$ of shedding vortices from a bluff body is proportional to flow velocity $U$ and inversely proportional to representative length of the body $L$, using Strouhal number St. For a cylinder, St is known as 0.2. The relationship is described as follows.

$$
f=S t \frac{U}{L}[\mathrm{~Hz}]
$$

In case of the mirror body, the highest power spectra were numerically obtained for $80,100 \mathrm{~km} / \mathrm{h}$ which are $16.6 \mathrm{~Hz}$ and $20.5 \mathrm{~Hz}$, respectively. Figure 24 shows the relationship between the frequency of the highest power

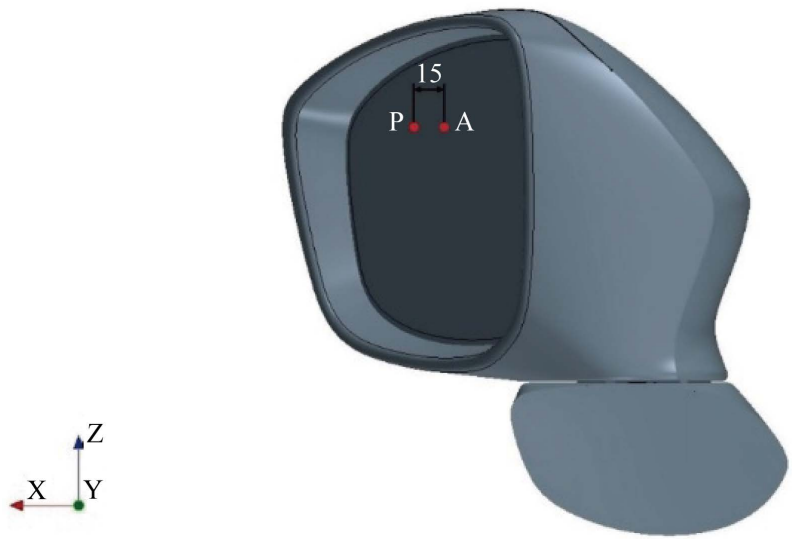

Figure 21. The measuring point $P$ is located $15 \mathrm{~mm}$ away vertically over the measuring point $A$ on the mirror surface.

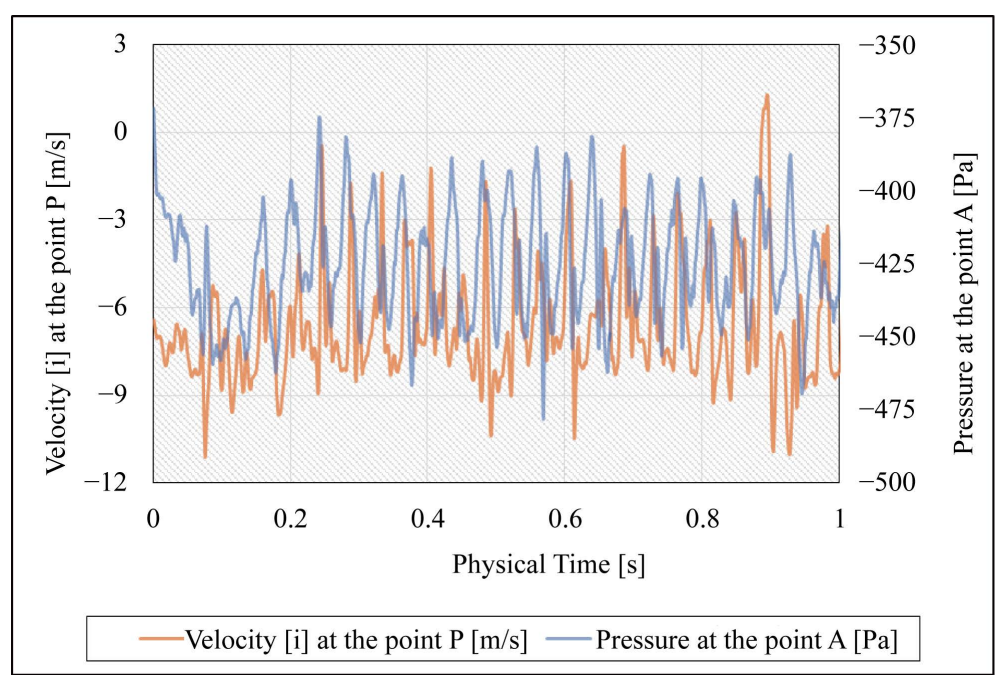

Figure 22. Relationship between flow velocity fluctuation at the point $P$ and pressure fluctuation at point $A$. 


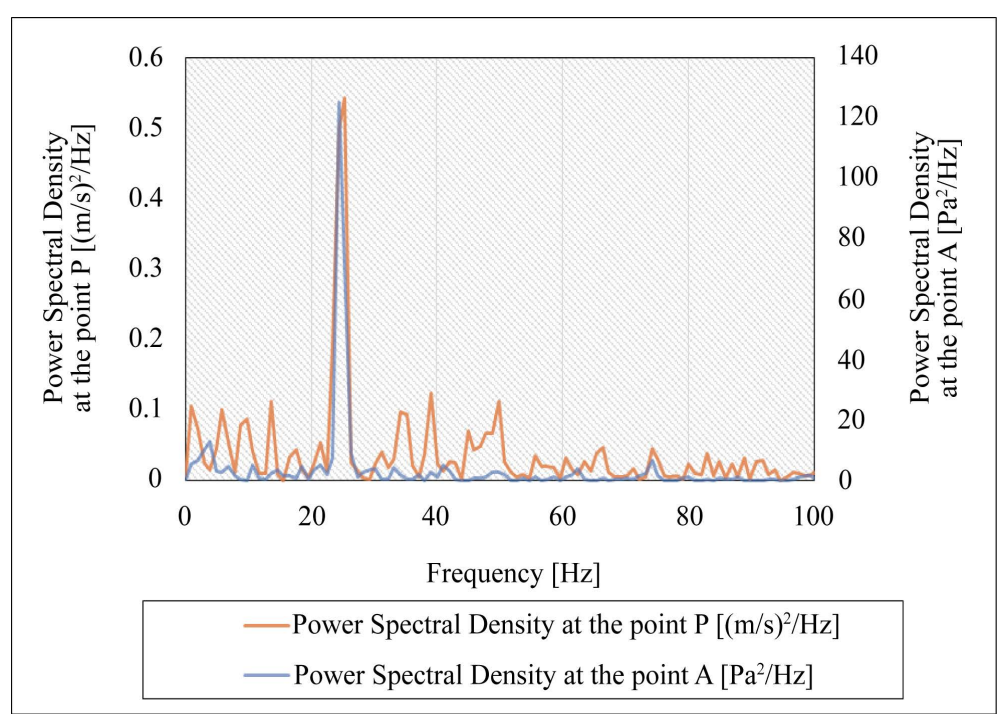

Figure 23. Power spectral densities on separation vortices at point $P$ and pressure fluctuation at point $A$ on the mirror surface at $120 \mathrm{~km} / \mathrm{h}(33.3 \mathrm{~m} / \mathrm{s})$.

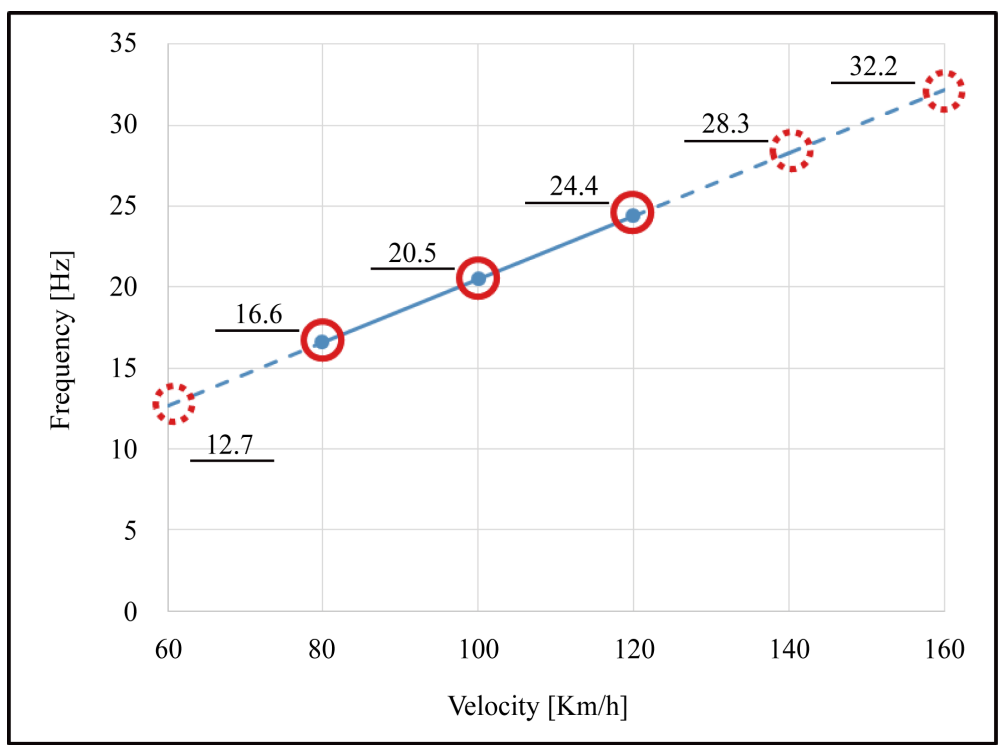

Figure 24. The relationship between flow velocities and peak frequencies obtained by FFT analyses for pressure fluctuations on the mirror surface.

spectra and flow velocity. Since $S t$ is calculated as 0.12 based on the graph, the frequencies of $140,160 \mathrm{~km} / \mathrm{h}$ are extrapolated as $28.3 \mathrm{~Hz}$ and $32.2 \mathrm{~Hz}$, respectively.

Experimental measurements reveal that the mirror has primary natural frequencies of 25 and $33 \mathrm{~Hz}$ in the $\mathrm{X}$ direction whereas 25 and $30 \mathrm{~Hz}$ both in the $\mathrm{Y}$ and $\mathrm{Z}$ direction. The accelerations of the mirror vibrations coincide with these primary natural frequencies. Therefore, image blurs are caused by vibration phenomena, which can be interpreted as resonance phenomena that the mirror surface resonates with the frequencies of shedding vortices. Since numerical analyses clarify that the frequencies of shedding vortices are $24.4 \mathrm{~Hz}$ at $120 \mathrm{~km} / \mathrm{h}$ and $28.3 \mathrm{~Hz}$ at $140 \mathrm{~km} / \mathrm{h}$, it follows that the shedding vortices exert aerodynamic loads on the mirror surface periodically with frequencies of $24.4 \mathrm{~Hz}$ and $28.3 \mathrm{~Hz}$. One of the ways to reduce vibration amounts is to shift frequencies of the external forces induced periodically by shedding vortices to as higher frequency range as possible because vibration amounts decrease which are inversely proportional to the square of the resonance frequency according to Equation (1). 


\section{Conclusion}

The paper clarifies relationships between causes and effects of side-view mirror vibration by means of the experimental method and Computational Fluid Dynamics. Both natural frequencies and vibrations of the mirror were measured in the wind tunnel under the same condition. Natural frequencies of the mirror were measured by the impact hammer test with coherence function for reliability of the data. In the measurement of vibrations, a vibration pickup set on the center point of the mirror surface measured accelerations in three directions for $\mathrm{X}, \mathrm{Y}$, and $\mathrm{Z}$ ranging from 60 to $160 \mathrm{~km} / \mathrm{h}$ at every $20 \mathrm{~km} / \mathrm{h}$ steps. As a result, it was found that the frequencies of the mirror vibration coincided completely with its primary natural frequencies. On the other hand, unsteady air-flow analyses clarified relationships between flow velocity fluctuations close to the mirror surface and pressure fluctuations on the mirror surface. It was also found that the two power spectra had peak values at the same frequency. This shows that flow velocity fluctuations affect directly pressure fluctuations on the mirror surface. Shedding vortices are, therefore, considered to be direct input sources to the mirror surface. As a result, the following findings are clarified. One is that frequencies of the side-view mirror vibration coincide with its natural frequencies even if any aerodynamic inputs are applied on the mirror. The other is that mirror vibration modes shift from the lower to the higher order vibration mode, responding aerodynamic inputs increasing in proportion to flow velocity around the mirror. Therefore it follows that image blurs at high speed cruising are caused by resonance phenomena that the mirror surface resonates with the frequencies of shedding vortices around the mirror.

\section{References}

[1] Iwai, T., Ishikawa, K., Kuroda, K. and Yamazaki, T. (2010) Experimental Statistical Energy Analysis for Automotive Rearview Mirror in Running. Transactions of the JSME, 5, 189-190. (In Japanese)

[2] Kato, Y. (2001) Numerical Analysis of the Vibration caused by Fluid-Dynamic Force Exerted on a Door Mirror Surface. $R \& D$ Review of Toyota CRDL, 36, 58. (In Japanese)

[3] Horinouchi, N., Kato, Y., Yamada, T. and Ninomiya, O. (2003) Development of Estimation Technique for Flow Induced Vibration on External Rearview Mirror. SAE Paper \# 2003-01-2815. http://dx.doi.org/10.4271/2003-01-2815

[4] Kawate, T., Ogawa, S. and Omori, I. (2015) Numerical Simulation on Side-View Mirror Vibration Induced by Separating Vortices. 93th JSME Fluids Engineering Conference, Tokyo University of Science, 7-8 November 2015.

[5] Ogawa, S., Kawate, T. and Omori, I. (2014) Study on Vibration of Mirror Surface Induced by Separated Flow behind a Side View Mirror. 27th JSME Computational Mechanics Division Conference, Iwate University, 22-24 November 2014.

[6] Ogawa, S., Takeda, J., Kawate, T. and Omori, I. (2014) Study on Control of Automobile Side-View Mirror Surface. 11th JSME OPTIS 2014, Kanazawa University, 12-13 December 2014. 


\author{
Nomenclature \\ $\delta$ : Shake Amount (m) \\ $a$ : Acceleration of Mirror Vibration $\left(\mathrm{m} / \mathrm{s}^{2}\right)$ \\ $f$ : Frequency of Mirror Vibration (Hz) \\ $R e$ : Reynolds Number \\ $U$ : Flow Velocity (m/s) \\ $L$ : Representative Length (m) \\ $v$ : Kinematic Viscosity $\left(\mathrm{m}^{2} / \mathrm{s}\right)$ \\ $P_{m}$ : Pressure of Measuring Points on Mirror Surface (Pa) \\ $P_{\infty}$ : Pressure in the Uniform Flow $(\mathrm{Pa})$ \\ $\rho$ : Density of Fluid $\left(\mathrm{kg} / \mathrm{m}^{3}\right)$ \\ $U_{\infty}$ : Flow Velocity in the Uniform Flow $(\mathrm{m} / \mathrm{s})$ \\ $\omega$ : Vorticity $(1 / \mathrm{s})$ \\ St: Strouhal Number
}

